\title{
Transposition in Lactobacillus sakei: inactivation of a second lactocin $S$ operon by the insertion of IS1520, a new member of the IS3 family of insertion sequences
}

\author{
Morten Skaugen and Ingolf F. Nes
}

\begin{abstract}
Author for correspondence: Morten Skaugen. Tel: +47 64949466. Fax: +47 64941465. e-mail: morten.skaugen@ikb.nlh.no
\end{abstract}

Laboratory of Microbial Gene Technology, Agricultural University of Norway, PO Box 5051, $\mathrm{N}-1432$ As-NLH, Norway

\begin{abstract}
The analysis of spontaneous bacteriocin-negative mutants has led to the identification and characterization of a new, transpositionally active, insertion sequence of the IS3 family in the lactocin-S-producing Lactobacillus sakei strain L45. The element, which has been designated IS1520, is 1302 bp long with $10 \mathrm{bp}$ perfect inverted repeat ends and generates direct repeats of a trinucleotide of target sequence upon transposition to the lactocin $S$ locus. IS1520 encodes two consecutive, partially overlapping, major ORFs, which are frameshifted in a manner typical of the IS3 family. Despite a low overall DNA sequence similarity, the putative frameshifting region of IS1520 is highly similar to the corresponding region of IS1163, which is a related element previously shown to be active in $L$. sakei $L 45$.
\end{abstract}

Keywords: transposition, insertion sequence, lantibiotic

\section{INTRODUCTION}

Lactocin $\mathrm{S}$ was originally described as an antimicrobial activity produced by Lactobacillus sakei L45 (Mørtvedt \& Nes, 1990), and was later found (Mørtvedt et al., 1991; Skaugen et al., 1994) to belong to the lantibiotics (Schnell et al., 1988) subgroup of bacteriocins. Lactocin $S$ production is unstable in L. sakei $\mathrm{L} 45$, which segregates nonproducing derivatives with high frequency under standard laboratory cultivation conditions. While most of the nonproducing isolates have lost the $50 \mathrm{~kb}$ production plasmid, pCIM1, and are sensitive to lactocin S $\left(\mathrm{Bac}^{-} \mathrm{Imm}^{-}\right)$, approximately one-third retain immunity as well as a structurally altered producer plasmid (Mørtvedt \& Nes, 1990; Skaugen \& Nes, 1994). We have previously shown that, in the latter category of production strain variants, the inactivation (and altered structure of pCIM1) correlates with the transposition of the IS3-like insertion sequence IS1163 into the lactocin S (las) region of pCIM1, thus demonstrating transpositional activity in L. sakei for the first time (Skaugen et al., 1994). The mutations created by IS1163 insertion into the complex las operon are all nonpolar (Skaugen et al., 1997), i.e. insertions do not result in premature termination of transcription. Systematic site-directed

The EMBL accession number for the 151520 nucleotide sequence is AJ250958. mutagenesis in L45 is effectively prevented by the fact that this strain and its derivatives are virtually untransformable. The relatively high frequency of occurrence and nonpolar nature of IS1163 insertions mean that the continued screening for and analysis of spontaneous knock-out $\left(\mathrm{Bac}^{-} \mathrm{Imm}^{+}\right)$mutants is an acceptable alternative strategy to probe for essential genes in the las cluster. Using the previously reported ligation/PCR protocol (Skaugen et al., 1997; Skaugen \& Nes, 1994), we have mapped a number of IS1163 insertions, spanning most of the las region. However, although this method is normally a reliable tool for the precise localization of mutations, some of the isolated $\mathrm{Bac}^{-}$ $\mathrm{Imm}^{+}$mutants escaped identification by this protocol. Below we present data demonstrating that these new mutations are caused by the insertion of a new IS3-like insertion sequence, IS1520, which is similar to, but distinct from, IS1163.

\section{METHODS}

Bacterial strains and plasmids. Lactobacillus sakei strains and plasmids used in this study are listed in Table 1 . The strains were cultivated at $30^{\circ} \mathrm{C}$ in MRS (Difco) liquid medium without aeration, or on MRS agar (1.5\%).

Escherichia coli DH5 $\alpha$ was cultivated at $37^{\circ} \mathrm{C}$ in $\mathrm{LB}$ liquid medium with aeration or on LB agar. Ampicillin for selecting and propagating resistant bacteria was added to a final concentration of $100 \mu \mathrm{g} \mathrm{ml}^{-1}$. 
Table 1. L. sakei strains and plasmids described in this study

\begin{tabular}{|lll|}
\hline Strain & Plasmid content/structure & $\begin{array}{c}\text { Relevant } \\
\text { phenotype }\end{array}$ \\
\hline L45 & pCIM1, pCIM2 & $\mathrm{Bac}^{+} \mathrm{Imm}^{+}$ \\
L45-2.1 & pCIM1 & $\mathrm{Bac}^{+} \mathrm{Imm}^{+}$ \\
L45-5.3 & pCIM153 (pCIM1 las X::IS1520) & $\mathrm{Bac}^{-} \mathrm{Imm}^{+}$ \\
L45-5.7 & pCIM157 (pCIM1 lasX::IS1520) & $\mathrm{Bac}^{-} \mathrm{Imm}^{+}$ \\
L45-5.8 & pCIM158 (pCIM1 lasN::IS1520) & $\mathrm{Bac}^{-} \mathrm{Imm}^{+}$ \\
\hline
\end{tabular}

Plasmids were isolated using a standard alkaline lysis method (Birnboim \& Doly, 1979), with elevated lysozyme concentrations (final concentration $5 \mathrm{mg} \mathrm{m}^{-1}$ ) when isolating plasmids from L. sakei. Total DNA was isolated from cultures of L. sakei as described previously (Skaugen et al., 1994).

Restriction analysis and molecular cloning. Enzymes used in restriction analyses and cloning experiments were purchased from New England Biolabs, Promega or Boehringer Mannheim.

E. coli DH5 $\alpha$ was transformed by electroporation (Dower et al., 1988) using the Bio-Rad Gene Pulser. DNA molecular size markers (1 kb ladder) were purchased from Gibco-BRL.

Amplification and nucleotide sequence determination. DNA (0.1-10 ng template) was amplified using AmpliTaq polymerase and the Perkin Elmer GeneAmp 9600 or MJ Research PTC-100 thermocycler. Amplification typically proceeded through 35 cycles after a 3 min hot-start $\left(97^{\circ} \mathrm{C}\right)$. The polymerizing time $\left(72^{\circ} \mathrm{C}\right)$ was set to $1 \mathrm{~min} 30 \mathrm{~s}$ in all reactions, except when amplifying from ligation mixes, where the polymerizing time was set to $3 \mathrm{~min}$. The annealing temperature was set at the $T_{\mathrm{m}}$ value for the oligonucleotide having the lowest calculated melting point. Annealing time and denaturing time $\left(94^{\circ} \mathrm{C}\right)$ were set to $1 \mathrm{~min}$ in all reactions.

For RT-PCR analysis, total RNA from L. sakei was isolated as described previously (Skaugen et al., 1997) and treated with RQ1 DNase (Promega) according to the manufacturer's instructions. The first-strand cDNA synthesis was carried out in $20 \mu \mathrm{l} 1 \times \mathrm{AMV}$ (avian myeloblastosis virus) reaction buffer containing $1 \mu \mathrm{g}$ total RNA, 100 pmol oligonucleotide primer (antisense of the $5^{\prime}$ end of the downstream gene), $40 \mathrm{U}$ RNasin (Promega), $2 \mathrm{mM}$ each dNTP and 5 U AMV RT (Promega) at $42{ }^{\circ} \mathrm{C}$ for $1 \mathrm{~h}$. After this time, $80 \mu \mathrm{l}$ amplification mix [dNTP $(0.2 \mathrm{mM}$ final concn), forward primer (100 pmol), Taq buffer $(1 \times$ final) and Taq polymerase $(2.5 \mathrm{U})]$ was added and the complete reaction was subjected to a standard amplification program $\left[97^{\circ} \mathrm{C}\right.$ for $5 \mathrm{~min}$ (hot-start), 35 cycles of $94{ }^{\circ} \mathrm{C}$ for $30 \mathrm{~s}, 55^{\circ} \mathrm{C}$ for $30 \mathrm{~s}, 72^{\circ} \mathrm{C}$ for $1 \mathrm{~min}$ ] and analysed on agarose gels. A reaction where AMV RT had been omitted was included as a control in each experiment.

Nucleotide sequences were determined using the cycle sequencing protocol of Perkin Elmer and the ABI Prism 310 or 377 automatic sequencer, with purified PCR product or plasmid DNA as template.

DNA-DNA hybridization. Restricted DNA was separated on agarose gels and blotted to GeneScreen Plus membranes (DuPont) using the Vacugene blotting apparatus (LKB Pharmacia). Hybridization probes were labelled using the Boehringer Mannheim Random Primed DNA Labelling kit and $\left[\alpha^{-32} \mathrm{P}\right] \mathrm{dCTP} \quad\left(3000 \mathrm{Ci} \mathrm{mmol}^{-1} ; 111 \mathrm{TBq}^{\mathrm{mmol}}{ }^{-1}\right)$
(Amersham). Prehybridization, hybridization and washing conditions were as described previously (Church \& Gilbert, 1984; Skaugen \& Nes, 1994). Autoradiography was carried out using Cronex film (DuPont) and intensifying screen at $-85{ }^{\circ} \mathrm{C}$.

Nucleotide sequence analysis. Nucleotide sequence data were assembled using the ABI/Perkin Elmer Autoassembler software, and analysed using the UWGCG program package (version 8.0). Database searches were performed using BLAST (Altschul et al., 1990, 1997) with default settings at the NCBI WWW server.

\section{RESULTS}

\section{Identification and sequence determination of plasmid-inserted IS1520}

Initially, the three $\mathrm{Bac}^{-} \mathrm{Imm}^{+}$mutants L45-5.3, L45-5.7 and L45-5.8 were subjected to the same ligation-PCR analysis which had previously been used to map IS1163 insertions. Briefly, plasmid DNA was digested to completion by Sau3AI, ligated to BamHI-digested pGEM7Zf $(+)$, and the ligation mixture was used as template in a PCR reaction in which one of the amplification primers was derived from either end of IS1163 and the other from the vector. When this protocol failed to yield a PCR product, the mutant plasmids were digested with several restriction enzymes, some of which cut in the IS1163 sequence and some of which do not. The digests were compared to corresponding digests of wild-type and IS1163-containing mutant plasmids. The comparison (not shown) demonstrated that the isolates L45-5.3, L45-5.7 and L45-5.8, like the previously characterized $\mathrm{Bac}^{-} \mathrm{Imm}^{+}$mutants, harboured plasmids (pCIM153, pCIM157 and pCIM158, respectively) larger than pCIM1, but with restriction patterns inconsistent with IS1163 insertion. Mutant restriction (XbaI) fragments were cloned in E. coli, and the junctions between inserted DNA and flanking regions were sequenced

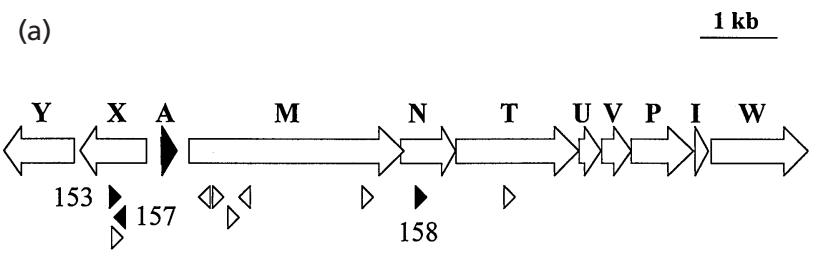

(b)

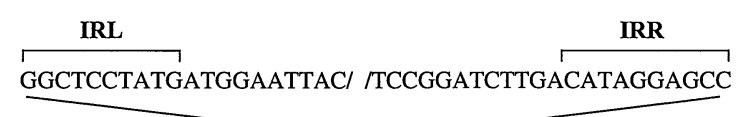

pCIM153 CCAAAATTGTTATGTGTTTC TTCATATATCGAAAGACTTT

pCIM157 TCAACCATTTATCAGCACTA CTATCACAAATTTACTATGA

pCIM158 TACCAGTGGTATTGACACAA CAATTAACGATGATATTCAA

Fig. 1. (a) Lactocin S locus, with the position and orientation of IS1163 and IS1520 insertions marked below with open and filled triangles, respectively. (b) Junction sequences of the three IS1520 insertions in the las cluster. The terminal nucleotides of each end of the element are shown at the top. 


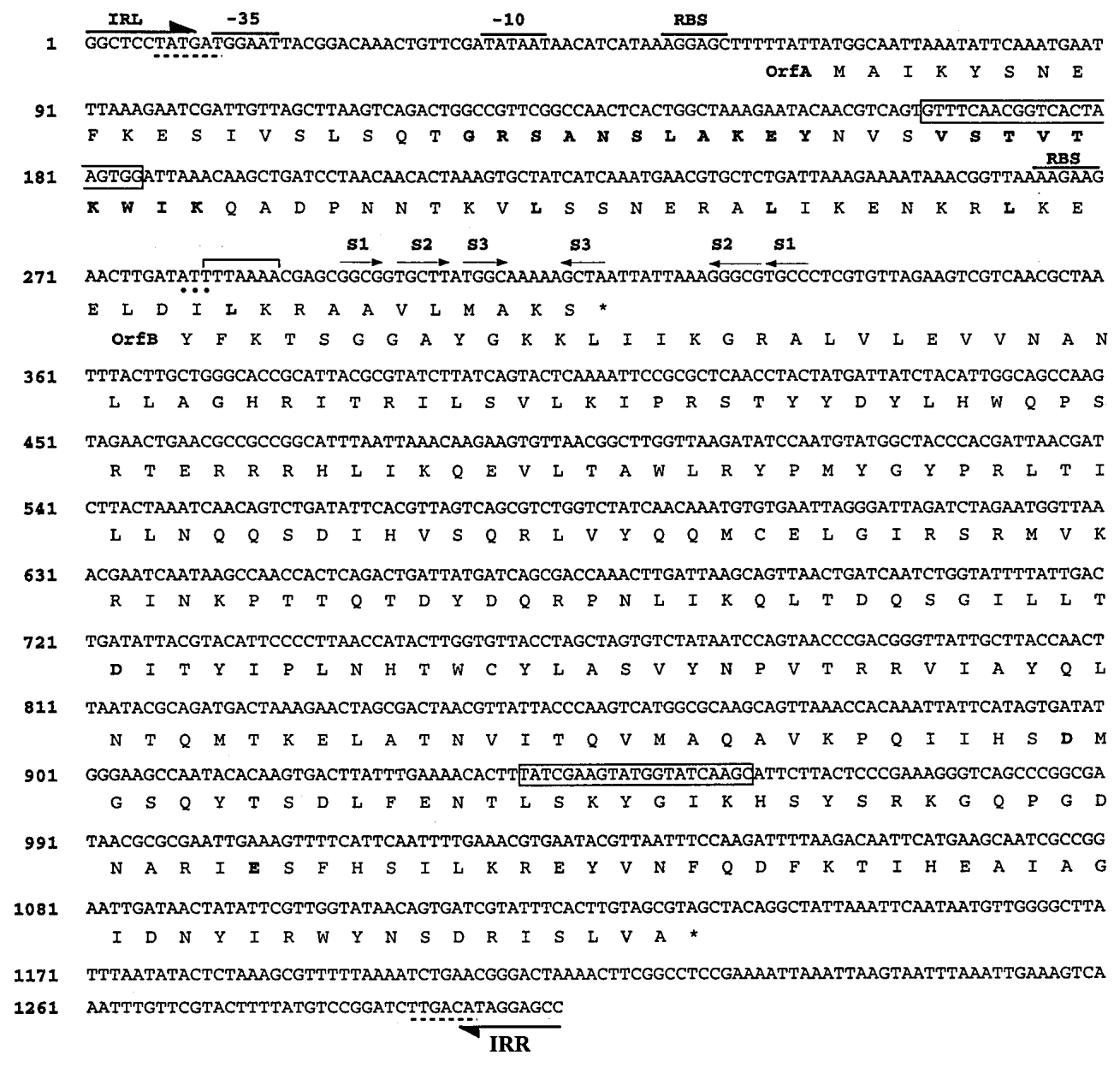

Fig. 2. Nucleotide sequence of IS1520, with the orfA and orfB translations shown below the coding sequence. The boxed nucleotides represent the sequences of the IS1520-specific primers used to amplify and sequence IS:pCIM1 junctions. The residues presumed to participate in the helix-turn-helix structure and the leucine residues of the putative leucine zipper in OrfA are indicated in bold, as are the conserved DD(35)E triad residues of OrfB. Putative ribosome-binding sites and the proposed promoter hexanucleotides $(-10$ and -35$)$ are overlined. The left and right terminal inverted repeats (IRL and IRR, respectively) are indicated by half-arrows. Also marked are the proposed frameshift region, including the tandem slippery codons (overlying square bracket), AUU codon (indicated by black dots) and nucleotides making up the stems of the putative downstream secondary structure (lettered arrows). The dashed lines represent the -10 (IRL) and -35 (IRR) elements of a possible promoter assembled by circularization of the element. See text for details.

using primers derived from the las region as well as the cloning vector. Subsequently, a primer pair complementary to the junctions in pCIM157 was designed, and the inserted DNA was amplified and sequenced in both directions. The complete nucleotide sequence was submitted to a database search, which yielded relatively weak but significant hits among the IS3 family of insertion sequences. pCIM153 and pCIM158 were shown to contain identical copies of the element, which was designated IS1520.

The GC content of IS 1520 is $37.6 \mathrm{~mol} \%$, which is similar to IS1163 (39 mol\%; Skaugen \& Nes, 1994), but significantly lower than the genomic value of 42-44 mol\% for L. sakei (Kandler \& Weiss, 1986). The GC content of the approximately $11 \mathrm{~kb}$ las cluster is $30 \cdot 2 \mathrm{~mol} \%$.

\section{Sequence analysis of IS1520}

Comparisons of nucleic acid and protein sequences suggest that IS1520 belongs to the IS3 subgroup (Mahillon \& Chandler, 1998) of the IS3 family, which is presently the largest and most widespread group of bacterial insertion sequences. IS1520 is 1302 bp long, has relatively short $(10 \mathrm{bp})$, perfect terminal inverted repeats (Fig. 2), and the plasmid insertions are flanked by (different) $3 \mathrm{bp}$ direct repeats (Fig. 1). Although IS1520 undoubtedly belongs to the IS3 family of insertion sequences, one conserved feature is absent: whilst nearly all elements in this group conform to the so-called 5'TG(/A) .... CA3' rule (Mahillon \& Chandler, 1998), IS1520 has G $\left(5^{\prime}\right)$ and $C\left(3^{\prime}\right)$ as its terminal residues (Fig. 2).

IS1520 contains two major ORFs, orf $A$ and $\operatorname{orf} B$, which 
overlap by $37 \mathrm{bp}$, and are probably co-transcribed from a promoter situated some $25 \mathrm{bp}$ upstream of the orf $A$ initiation codon (Fig. 2). The reading phases of orf $A$ and $\operatorname{orf} B$ are 0 and -1 , respectively, which is an organization found in most IS3-related elements. Despite a low overall sequence similarity $(52 \%$, in a GAP comparison containing 10 gaps) between IS1163 and IS1520, the putative frameshift regions have almost $77 \%$ identity (no gaps) in a 65 bp window (nt 246-310; Fig. 2) containing the putative ribosome-binding sites, the so-called 'tandem slippery codons' (Atkins et al., 1990) constituted by the heptanucleotide $5^{\prime}$-UUUAAAA- $3^{\prime}$, and the $5^{\prime}$ part of the first stem in both the proposed downstream secondary structures.

As is the case for many of its relatives (Mahillon \& Chandler, 1998), the IS1520 OrfA contains a relatively strong helix-turn-helix motif in the N-terminal half of the protein, and a leucine zipper motif towards the Cterminus (Fig. 2). The function of the former motif is probably the specific recognition of the element's end(s) by OrfAB as well as OrfA (Hu et al., 1996; Lei \& Hu, 1997; Ton-Hoang et al., 1998), while the leucine zipper is likely to promote the transposase oligomerization required for transposition (Haren et al., 1998; Lei \& Hu, 1997). A modulatory role for OrfA in transposition has been demonstrated for at least two members of the IS3 family [IS911 (Polard et al., 1992) and IS3 (Sekine et al., 1994)], and the IS2 OrfA has been shown to affect orf $A / B / A B$ transcription by binding to the promoter overlapping the left inverted repeat of this element (Hu et al., 1996).

orf $B$ encodes the C-terminal two-thirds of the putative IS1520 OrfAB transposase, which constitutes the catalytic domain of the protein with the highly conserved DD(35)E triad (Doak et al., 1994). There are no combinations of a proper ribosome-binding site and a standard initiation codon associated with the IS1520 orfB, suggesting that OrfB may not be produced as a separate protein. However, as in IS1163, there is an AUU codon, in reading frame $0,8 \mathrm{nt}$ downstream of a putative ribosome-binding site and overlapping the proposed heptanucleotide frameshifting site (Fig. 2). For the related element IS911, it has been demonstrated that a similarly situated AUU codon, in reading frame -1 , is utilized as the OrfB initiation codon (Polard et al., 1991).

The presence of a perfect consensus -35 box hexanucleotide (nt 1289-1294; Fig. 2) partially overlapping the right inverted terminal repeat suggests that IS1520 could participate in the generation of a hybrid promoter upon insertion, although analysis of the junction sequences created by the insertions failed to identify any such promoters. For at least some of the IS3 family members (Mahillon \& Chandler, 1998) it has been demonstrated that the element can excise as a circle in which the left and right ends are joined via a short spacer, which for IS911 corresponds to one of the direct repeats flanking the element in the donor location (Polard et al., 1992). In the case of IS911, it has been shown that this circularization assembles a strong promoter that could ensure the high levels of transposase

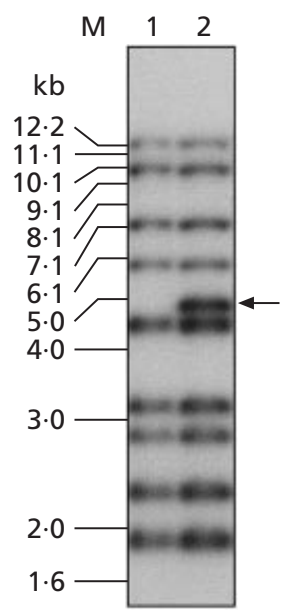

Fig. 3. Minimal genomic copy number estimation of IS1520. The element was amplified from pCIM157 and used to probe restricted (EcoRI and HindIII) total DNA isolated from L45-2.1 (lane 1) and the IS1520-generated lasX mutant L45-5.7 (lane 2). The arrow indicates the position of the pCIM157 fragment containing the new copy of IS1520. The approximate positions of the 12 larger bands of the molecular size marker (sizes ranging from 1.6 to $12.2 \mathrm{~kb}$ ) are shown on the left (M).

required for the circles to function efficiently as substrates for the subsequent insertion reaction (TonHoang et al., 1997). Although it is uncertain whether the circularization and hybrid promoter assembly is a general feature of the IS3 family, it is interesting to note that the circularization of IS1520 with a $3 \mathrm{bp}$ spacer between the repeats would place the IRR -35 element at a distance of $17 \mathrm{bp}$ from the near perfect -10 hexanucleotide TATGAT in the IRL, thus creating an at least theoretically strong promoter of orf $A$ and $B$ transcription.

\section{Genomic copy number of IS1520 in L45}

To determine the genomic copy number of IS1520, the element was amplified from pCIM157, labelled and used to probe a Southern blot of HindIII- and EcoRI-digested total DNA from various isolates of L45 as well as nonlactocin-S-producing strains from our laboratory collection. The result of one such hybridization is shown in Fig. 3 and indicates a minimum genomic copy number of 9 in L45. All L. sakei strains tested had fragments hybridizing to the IS1520 probe, with a mean (minimum) copy number of $2-3$ (results not shown).

\section{Effects of IS1520 insertion on lactocin S expression}

The three IS1520 insertions disrupt either of two las cluster genes (Fig. 1), both of which are novel in a lantibiotic context. The pCIM158 insertion is located in the $5^{\prime}$ third of las $N$, which encodes a putative $27 \cdot 1 \mathrm{kDa}$ protein previously designated Orf239 (Skaugen et al., 1997). Both pCIM153 and pCIM157 have IS1520 inserted in las X, which is the proximal gene in a dicistronic operon located upstream of las $A-W$ and transcribed in the opposite direction (M. Skaugen and 


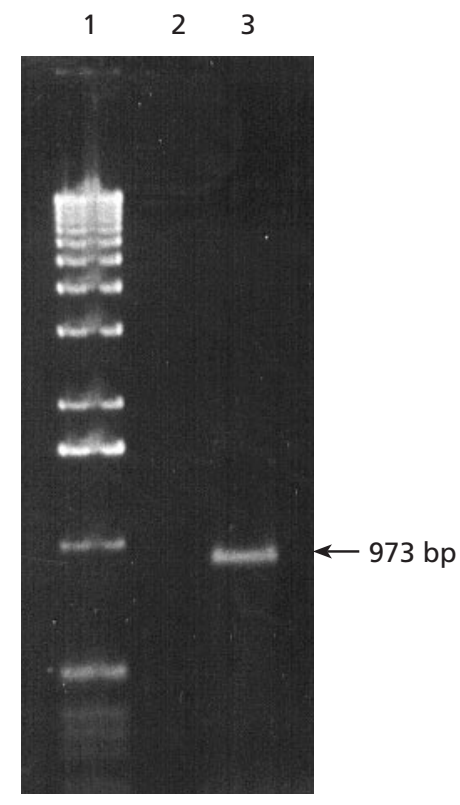

Fig. 4. RT-PCR using RNA isolated from IS1520-generated las $X$ mutant L45-5.7. The oligonucleotide used for first-strand CDNA synthesis was the lasY-specific oligonucleotide (see Methods), which was complemented with the downstream of the two primers indicated in Fig. 2 for second-strand synthesis and amplification. Lanes: 1 , molecular size marker; 2 and 3, 1/10 of each of two RT-PCR reactions including (lane 3 ) or omitting (lane 2) AMV RT. The PCR product (arrow) has the expected size (973 bp) and represents RNA reading into the downstream ORF (lasY) through (or from within) IS1520.

others, unpublished results). las X encodes a 285 residue polypeptide with weak similarity to Rgg, a regulator of gtfG expression in Streptococcus gordonii (Sulavik et al., 1992; Sulavik \& Clewell, 1996).

Complementation of the insertion mutations by reintroducing the affected gene cloned in a suitable vector is, in practice, prevented by the difficulties with the transformation of L45. Therefore, to test for polarity of the insertions (i.e. premature termination of transcription), RT-PCR experiments were set up using RNA isolated from the mutants (see Methods). Antisense primers for either of the downstream genes (las T, $5^{\prime}$ ATGCATACTTAGTCGTCTCC-3'; or las Y, 5'-TCCACACTAGATTAGCACAGAAG-3') were used for first-strand cDNA synthesis, and were complemented with either of the two IS1520-specific primers indicated in Fig. 2 for the PCR step. RNA-specific amplification was achieved in all three cases (shown for L45-5.7 in Fig. 4), indicating that the IS1520 insertions described here do not have polar effects.

\section{DISCUSSION}

Immune nonproducers $\left(\mathrm{Bac}^{-} \mathrm{Imm}^{+}\right)$can be isolated from a L. sakei L45 culture at a frequency of approximately one per 1000 c.f.u., and we have previously reported that insertion of the IS3-like element IS1163 into pCIM1 is one cause of this phenotype. We have shown here that transposition of IS1520, which is related to, but distinct from, IS1163, contributes to the frequent occurrence of these mutants. For the three $\mathrm{Bac}^{-} \mathrm{Imm}^{+}$ isolates examined, there is a correlation between the presence of a copy of IS1520 in las N in the previously described las $A-W$ operon or las $X$ in the divergently transcribed las XY operon and the abolition of lactocin $S$ production, and we therefore conclude that IS1520 insertion causes the cessation of production in these cases.

The simplest explanation for the observed inactivation of the las operon is that insertion results in aborted translation of the target ORF, indicating a role for the affected gene/gene product in lactocin $S$ production. The polarity tests performed support this conclusion, although we cannot at this point exclude the possibility that outward transcription from the element may at least contribute to these results.

As is the case with the related, co-resident element IS1163, the identified IS1520 insertion sites have no obvious common features suggesting any target specificity of insertion. Such specificity could, however, be of a regional kind, which is not easily identified by sequence analysis. It is worth noting in this context that the GC content of the pCIM1 region to which all IS1520 insertions have been mapped is $29 \cdot 8 \mathrm{~mol} \%$. This value is significantly lower than the estimated genomic value of $43 \mathrm{~mol} \%$ for L. sakei (Kandler \& Weiss, 1986), thereby indicating a possible preference of transposition to ATrich regions (Craig, 1997).

The sequence and genetic organization of IS1520 place the element in the IS3 family of bacterial insertion sequences (Mahillon \& Chandler, 1998). This group is probably the most widespread of all IS elements, and at least three distinct members of the IS3 family have previously been identified in the genus Lactobacillus (Shimizu-Kadota et al., 1985; Skaugen \& Nes, 1994; Walker \& Klaenhammer, 1994).

For the IS3-type elements where the issue has been addressed experimentally, it has been shown that a frameshift event fusing the OrfA and OrfB proteins into the transposase, OrfAB, is required for transposition (Polard et al., 1991; Sekine et al., 1994; Vögele et al., 1991). This so-called programmed frameshifting (Chandler \& Fayet, 1993) occurs at a specific 'slippery' heptanucleotide in the overlap region and its efficacy is, at least in some cases, modulated by an upstream ShineDalgarno-like sequence and/or a downstream secondary structure of varying complexity (Rettberg et al., 1999; Sekine et al., 1994; Vögele et al., 1991). The dependence on frameshifting for the expression of essential genes is a feature the IS3 family shares with retroviruses as well as many bacterial and bacteriophage genes (Atkins et al., 1990; Fayet et al., 1990), and the model for ribosomal rephasing was originally proposed to account for the presence of transframe proteins in retroviral systems (Jacks et al., 1988).

Structures in IS1520 which may be of significance in the expression of the transposase genes are suggested by 
sequence alignments, and the IS1520 frameshift heptanucleotide thus indicated has the sequence $5^{\prime}$ UUUAAAA-3', which is identical to that proposed for IS1163 (Skaugen \& Nes, 1994). Downstream of these tandem slippery codons is a region which could form a stem-loop structure masking the stop codon of orf $A$, and a Shine-Dalgarno motif is found upstream at a distance indicating a role in frameshifting and possibly in initiating translation of orf $B$. The AUU codon, which is the initiation codon of the IS911 orfB (Polard et al., 1991), is present in both IS1163 and IS1520, but in frame 0 rather than -1 . The use of this codon in initiating translation of $\operatorname{orf} B$ would therefore require a -1 shift of reading phase immediately after initiation, an event which has been shown to be a prerequisite for translation of the second ORF (insB) of IS150 (Vögele et al., 1991).

The low overall sequence similarity between IS1520 and IS1163 suggests that they have entered their common host as distinct elements. The high similarity between the two elements' proposed frameshifting regions may therefore reflect independent adaptations to the host rather than conservation of a common original sequence. Whichever is true, the similarity suggests functional significance of the sequence motifs in the region, thus adding to the diversity of this particular kind of gene expression regulator. It should be noted at this point that the suggested functional regions of IS1520 lack experimental support, and that the significance of the features discussed above with respect to the IS1520 transpositional activities remains to be determined. The results reported here should provide a basis for future experiments addressing these basic issues as well as for the continued exploitation of a natural transposon mutagenesis system in L. sakei.

\section{ACKNOWLEDGEMENTS}

Part of this work was supported by grant 114322/112 from the Norwegian Research Council.

\section{REFERENCES}

Altschul, S. F., Gish, W., Miller, W., Myers, E. W. \& Lipman, D. J. (1990). Basic local alignment search tool. J Mol Biol 215, 403-410.

Altschul, S. F., Madden, T. L., Schaffer, A. A., Zhang, J., Zhang, Z., Miller, W. \& Lipman, D. J. (1997). Gapped BLAST and PSI-BLAST : a new generation of protein database search programs. Nucleic Acids Res 25, 3389-3402.

Atkins, J. F., Weiss, R. B. \& Gesteland, R. F. (1990). Ribosome gymnastics - degree of difficulty 9.5, style 10·0. Cell 62, 413-423.

Birnboim, H. C. \& Doly, J. (1979). A rapid alkaline extraction procedure for screening recombinant plasmid DNA. Nucleic Acids Res 7, 1513-1523.

Chandler, M. \& Fayet, O. (1993). Translational frameshifting in the control of transposition in bacteria. Mol Microbiol 7, 497-503.

Church, G. M. \& Gilbert, W. (1984). Genomic sequencing. Proc Natl Acad Sci USA 81, 1991-1995.

Craig, N. L. (1997). Target site selection in transposition. Annu Rev Biochem 66, 437-474.
Doak, T. G., Doerder, F. P., Jahn, C. L. \& Herrick, G. (1994). A proposed superfamily of transposase genes: transposon-like elements in ciliated protozoa and a common "D35E" motif. Proc Natl Acad Sci USA 91, 942-946.

Dower, W. J., Miller, J. F. \& Ragsdale, C. W. (1988). High efficiency transformation of E. coli by high voltage electroporation. Nucleic Acids Res 16, 6127-6145.

Fayet, O., Ramond, P., Polard, P., Prére, M. F. \& Chandler, M. (1990). Functional similarities between retroviruses and the IS3 family of bacterial insertion sequences? Mol Microbiol 4, 1771-1777.

Haren, L., Polard, P., Ton-Hoang, B. \& Chandler, M. (1998). Multiple oligomerisation domains in the IS911 transposase: a leucine zipper motif is essential for activity. J Mol Biol 283, 29-41.

Hu, S. T., Lee, L. C. \& Lei, G. S. (1996). Detection of an IS2-encoded 46-kilodalton protein capable of binding terminal repeats of IS2. J Bacteriol 178, 5652-5659.

Jacks, T., Madhani, H. D., Masiarz, F. R. \& Varmus, H. E. (1988). Signals for ribosomal frameshifting in the Rous sarcoma virus gag-pol region. Cell 55, 447-458.

Kandler, O. \& Weiss, N. (1986). Regular, nonsporing Grampositive rods. In Bergey's Manual of Systematic Bacteriology, pp. 1208-1260. Edited by P. A. Sneath, N. S. Mair, M. E. Sharpe \& J. G. Holt. Baltimore: Williams \& Wilkins.

Lei, G. S. \& Hu, S. T. (1997). Functional domains of the InsA protein of IS2. J Bacteriol 179, 6238-6243.

Mahillon, J. \& Chandler, M. (1998). Insertion sequences. Microbiol Mol Biol Rev 62, 725.

Mørtvedt, C. I. \& Nes, I. F. (1990). Plasmid-associated bacteriocin production by a Lactobacillus sake strain. J Gen Microbiol 136, 1601-1607.

Mørtvedt, C. I., Nissen-Meyer, J., Sletten, K. \& Nes, I. F. (1991). Purification and amino acid sequence of lactocin $S$, a bacteriocin produced by Lactobacillus sake L45. Appl Environ Microbiol 57, 1829-1834.

Polard, P., Prére, M. F., Chandler, M. \& Fayet, O. (1991). Programmed translational frameshifting and initiation at an AUU codon in gene expression of bacterial insertion sequence IS911. J Mol Biol 222, 465-477.

Polard, P., Prére, M. F., Fayet, O. \& Chandler, M. (1992). Transposase-induced excision and circularization of the bacterial insertion sequence IS911. EMBO J 11, 5079-5090.

Rettberg, C. C., Prére, M. F., Gesteland, R. F., Atkins, J. F. \& Fayet, O. (1999). A three-way junction and constituent stem-loops as the stimulator for programmed -1 frameshifting in bacterial insertion sequence IS911. J Mol Biol 286, 1365-1378.

Schnell, N., Entian, K.-D., Schneider, U., Götz, F., Zähner, H., Kellner, R. \& Jung, G. (1988). Prepeptide sequence of epidermin, a ribosomally synthesized antibiotic with four sulphide-rings. Nature 333, 276-278.

Sekine, Y., Eisaki, N. \& Ohtsubo, E. (1994). Translational control in production of transposase and in transposition of insertion sequence IS3. J Mol Biol 235, 1406-1420.

Shimizu-Kadota, M., Kiwaki, M., Hirokawa, H. \& Tsuchida, N. (1985). ISL1: a new transposable element in Lactobacillus casei. Mol Gen Genet 200, 193-198.

Skaugen, M. \& Nes, I. F. (1994). Transposition in Lactobacillus sake and its abolition of lactocin $\mathrm{S}$ production by insertion of IS1163, a new member of the IS3 family. Appl Environ Microbiol 60, 2818-2825.

Skaugen, M., Nissen-Meyer, J., Jung, G., Stefanovic, S., Sletten, 
K., Abildgaard, C. I. M. \& Nes, I. F. (1994). In vivo conversion of Lserine to D-alanine in a ribosomally synthesized polypeptide. $J$ Biol Chem 269, 27183-27185.

Skaugen, M., Abildgaard, C. I. M. \& Nes, I. F. (1997). Organization and expression of a gene cluster involved in the biosynthesis of the lantibiotic lactocin S. Mol Gen Genet 253, 674-686.

Sulavik, M. C. \& Clewell, D. B. (1996). Rgg is a positive transcriptional regulator of the Streptococcus gordonii gtfG gene. J Bacteriol 178, 5826-5830.

Sulavik, M. C., Tardif, G. \& Clewell, D. B. (1992). Identification of a gene, $r g g$, which regulates expression of glucosyltransferase and influences the Spp phenotype of Streptococcus gordonii challis. J Bacteriol 174, 3577-3586.

Ton-Hoang, B., Betermier, M., Polard, P. \& Chandler, M. (1997).
Assembly of a strong promoter following IS911 circularization and the role of circles in transposition. EMBO J 16, 3357-3371.

Ton-Hoang, B., Polard, P. \& Chandler, M. (1998). Efficient transposition of IS911 circles in vitro. EMBO J 17, 1169-1181.

Vögele, K., Schwartz, E., Welz, C., Schiltz, E. \& Rak, B. (1991). High-level ribosomal frameshifting directs the synthesis of IS150 gene products. Nucleic Acids Res 19, 4377-4385.

Walker, D. C. \& Klaenhammer, T. R. (1994). Isolation of a novel IS3 group insertion element and construction of an integration vector for Lactobacillus spp. J Bacteriol 176, 5330-5340.

Received 16 November 1999; revised 4 February 2000; accepted 10 February 2000. 